\title{
Exceptional response to radiotherapy in unresectable pleuropulmonary blastoma of a child
}

\author{
Jae Sik Kim, Joo Ho Lee \\ Department of Radiation Oncology, Seoul National University Hospital, Seoul, Korea
}

Received: May 19, 2020

Revised: June 5, 2020

Accepted: June 12, 2020

Correspondence:

Joo Ho Lee

Department of Radiation Oncology,

Seoul National University Hospital,

101 Daehak-ro, Jongno-gu, Seoul

03080, Korea

Tel: +82-2-2072-2819

Fax: +82-2-765-3317

E-mail: jhlee@snuh.org

ORCID:

https://orcid.org/0000-0001-7248-3214
Pleuropulmonary blastoma (PPB) is a rare intrathoracic neoplasm in children. Although surgery with or without chemotherapy mainly conducted, the response of radiotherapy (RT) has not been evaluated yet. For unresectable tumor, RT might be considered as one option to decrease tumor extent to relieve obstructing symptoms or to facilitate successive treatment. We report one child in whom PPB with DICER1 mutation recurred after surgery and lead to respiratory distress. She emergently received palliative RT with a relatively low dose (20 Gy), and symptoms sufficiently relieved. Even she showed an $84.3 \%$ reduction in diameter and maintained the remission status for 1 year. These might reflect possible radiosensitivity of $\mathrm{PPB}$, and further investigations of RT might be necessary for unresectable PPB.

Keywords: Child, Radiotherapy, Pleuropulmonary blastoma

\section{Introduction}

Pleuropulmonary blastoma (PPB) is a rare neoplasm that occurs mainly in children and arises from the lung or pleura, with highly aggressive features [1]. Clinically, PPB presents with non-specific symptoms, commonly including respiratory distress, fever, and chest pain. Although there is currently no standard treatment guideline for PPB, mainly surgical resection and chemotherapy, if necessary, are performed [2]. However, the role of radiotherapy (RT) is unclear to date, and while one case report described a response to RT for unresectable PPB in an adult [3], the response and efficacy of RT in treating gross residual PPB has not been reported in pediatric patients who account for approximately $45.5 \%$ of patients with PPB [4]. In this report, we present one case showing the exceptional response to short-course RT for respiratory failure due to recurred PPB with DICER1 mutation in a child.

\section{Case Report}

A 6-year-old girl who had received right upper lobectomy for type II PPB in May 2015 presented fever, cough, sputum, and dyspnea from 10 days ago. She was prescribed medicine for upper respiratory infection in another hospital. Symptoms did not improve, and she was referred to the emergency department for right lung total collapse on chest X-ray of another hospital with tachypnea and wheezing on April 26, 2019. Chest computed tomography (CT) found newly noted about $22.3 \times 13.2 \times 12.7 \mathrm{~cm}^{3}$ sized cystic and solid mass in the right hemithorax (Fig. 1A). After a disease-free interval of 4 years, PPB recurred with rapid progression. In echocardiography on April 27, 2019, both atria collapsed, and compressed superior and inferior vena cava were observed. IVADo regimen (ifosfamide, vincristine, actinomycin D, and doxorubicin) was performed to relieve the vascular obstruction from April 28, 2019. 

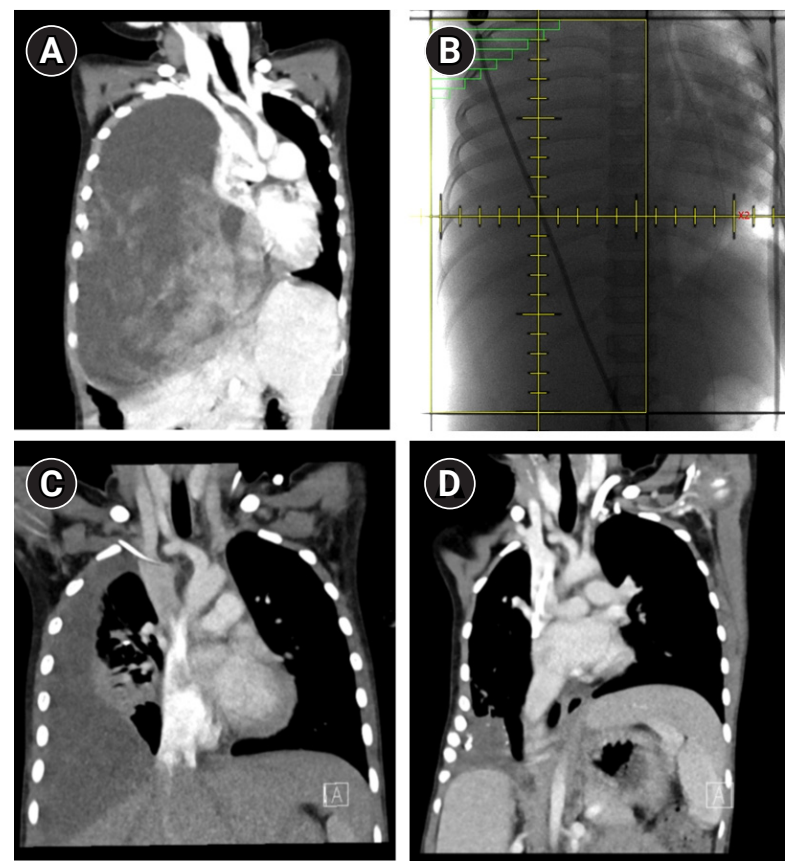

Fig. 1.Contrast-enhance chest computed tomography scans: (A) before treatment, (B) radiotherapy field of patient, (C) 1 month after radiotherapy, and (D) at last follow-up.

However, 2 days after chemotherapy, she was transferred to a pediatric intensive care unit for applying mechanical ventilator due to respiratory failure and obstructive shock with tumor progression. For respiratory symptom relief, emergency RT was delivered from April 30 to May 3, 2019. RT to right lung mass was given with anteroposterior/posteroanterior field $\left(11 \times 20 \mathrm{~cm}^{2}\right)$ weighted $1: 1$ to a total dose of $20 \mathrm{~Gy}$ in 4 fractions using $10 \mathrm{MV}$ photon (Fig. 1B). Echocardiography on May 8, 2019 found no more compression of right atrium and superior vena cava. One week after RT, extubation was done and no oxygen desaturation observed. However, because she presented dyspnea, oxygen was supplied via nasal prong (up to $4 \mathrm{~L} / \mathrm{min}$ ) or facial mask (up to $7 \mathrm{~L} / \mathrm{min}$ ). Ten days later, her blood-oxygen saturation level $\left(\mathrm{SpO}_{2}\right)$ was $97 \%$ in room-air and nasal prong was used intermittently. After one month without any further chemo- or radiotherapy, the cystic and solid mass in the right hemithorax markedly reduced to $6.5 \times 6.2 \times 5.3 \mathrm{~cm}^{3}$ in chest CT (Fig. 1C). She had remained in stable respiratory status in room-air and could re-started 4 cycles of IVADo. PPB continued to decrease and was measured up to $3.5 \times 3.1 \times 2.5 \mathrm{~cm}^{3}$ in the last follow-up chest CT (9 months after RT) (Fig. 1D). From December 2019, pulse VAC (vincristine, actinomycin D, and cyclophosphamide) treatment started. After 4 cycles of chemotherapy, needle biopsy of residual tumor on March 31, 2020 found no tumor, only fibrous tissues. In this patient, next-generation sequencing analysis using a previous surgical sample detected DICER 1 mutation (c. $5125 \mathrm{G}>\mathrm{A}$ ).
This study was approved by the Institutional Review Board of Seoul National University Hospital (IRB No. H-1907-118-1048). Because of the retrospective review of the case and excluding all patient identifiers in manuscript and figures, the requirement for obtaining informed consent of patient in this report was waived.

\section{Discussion}

PPB is an extremely rare and distinct primary malignant neoplasm of lung and pleura, accounting for $0.5 \%$ of all malignant neoplasms in the pediatric population $[5,6]$. In the early stage of tumorigenesis, the epithelium of airspace expands and forms the cyst. Mesenchymal cells undergoing malignant transformation show sarcomatous overgrowth, finally producing solid masses [7]. Depending on where neoplasm is in the tumorigenesis stage, PPB is divided into three subtypes based on its morphological features [5]. Type I is purely cystic with subtle malignant transformation, typically occurring in very young children under about 2 years old. If type I PPB has no primitive cell component, it is designated as type Ir (the " $r$ " means regression or non-progression) [8]. Type I could progress to type II or III, but not all. Hill et al. [7] reported that among 51 patients with type I PPB, 5 patients experienced local recurrence or disease progression. Of 4 patients with available microscopic sections of the recurred PPB, 2 progressed to type II, and the others showed type III pattern. Type II and type III exhibit similar solid components, and type II PPB has residual cystic areas. In type II and type III, relapse at the central nervous system with or without local recurrence is the major failure pattern (59.1\%) [8]. The 5-year overall survival rate for type I, II, and III was 89\%-100\%, 67\%$71 \%$, and 53\%-67\%, respectively [2,9]. This result emphasizes that type I PPB should be prevented from progressing to type II and III.

Currently, there is no standard treatment, but the International Pleuropulmonary Blastoma Registry recommends general treatment options. The treatment of choice for patients with type I and Ir PPB is complete surgical resection. In these patients, adjuvant chemotherapy does not affect the progression of the disease and patient survival. For patients with type II and III PPB, a multimodal approach is recommended, including chemotherapeutic regimens for rhabdomyosarcoma before or after surgery [2]. In terms of RT, previous reports did not show a survival benefit $[8,10]$. However, although experience with one patient with recurrent PPB is inconclusive, even low dose short-course RT consisting of 20 Gy could rapidly reduce the size of PPB in emergencies such as respiratory failure. Accumulation of clinical experiences about the use of RT is needed.

DICER1 gene has a critical regulatory role in the generation of microRNA [11]. Moreover, DICER1 has been known to play an es- 
sential role in lung morphogenesis [12]. Messinger et al. [8] found that $66 \%$ of patients with PPB possessed a heterozygous, deleterious mutation of DICER1. There were no differences in both clinical features and prognosis between patients harboring a DICER1 mutation and those not. In that report, only 20\% of patients ( $n=47)$ with type II and III PPB were treated with RT, and the prognostic significance for a DICER1 mutation in patients receiving RT was not analyzed.

Several in vitro experiments have reported that DICER is associated with DNA damage response (DDR) [13-15]. DNA damage response RNAs are generated by DICER and DROSHA [13], and these recruit DDR factors to the DNA lesions [14]. It has also been demonstrated that the depletion of DICER1 results in endogenous DNA damage and delay of DDR [15]. Considering that RT causes tumor cell death by DNA damage, DICER1 mutation of PPB might be closely related to increased radiosensitivity. Comprehensive biologic researches on radiosensitivity and DICER1 would be required. Furthermore, because most patients with PPB have DICER1 mutation, it could be expected as a target gene of RT.

In conclusion, PPB is an aggressive intrathoracic neoplasm with poor prognosis in early childhood. Multimodality approaches to treat PPB, including surgery, chemotherapy, and RT, should be considered. Our experience suggests that PPB might have high radiosensitivity.

\section{Conflict of Interest}

No potential conflict of interest relevant to this article was reported.

\section{References}

1. Manivel JC, Priest JR, Watterson J, et al. Pleuropulmonary blastoma. The so-called pulmonary blastoma of childhood. Cancer 1988;62:1516-26.

2. National Cancer Institute. PDO Cancer Information Summaries [Internet]. Bethesda MD: National Cancer Institute; 2002 [cited 2020 Jun 14]. Available from: https://www.ncbi.nlm.nih.gov/ books/NBK82221/.

3. Kamenova B, Braverman AS, Axiotis CA, Sohn C, Goff DJ. Complete remission of an unrectable pleuropulmonary blastoma in an adult after radiation therapy. Am J Clin Oncol 2006;29:641-2.

4. Indolfi P, Casale F, Carli M, et al. Pleuropulmonary blastoma: management and prognosis of 11 cases. Cancer 2000;89:1396401.

5. Dehner LP. Pleuropulmonary blastoma. a unique intrathoracic pulmonary neoplasm of childhood. Perspect Pediatr Pathol 1995; 18:214-6.

6. Sengupta S, Chatterjee U, Bandyopadhyay R, Bhowmick K, Banerjee S. Primary pulmonary neoplasms in children: a report of five cases. Indian J Med Paediatr Oncol 2011;32:223-6.

7. Hill DA, Jarzembowski JA, Priest JR, Williams G, Schoettler $P$, Dehner LP. Type I pleuropulmonary blastoma: pathology and biology study of 51 cases from the international pleuropulmonary blastoma registry. Am J Surg Pathol 2008;32:282-95.

8. Messinger $\mathrm{YH}$, Stewart DR, Priest JR, et al. Pleuropulmonary blastoma: a report on 350 central pathology-confirmed pleuropulmonary blastoma cases by the International Pleuropulmonary Blastoma Registry. Cancer 2015;121:276-85.

9. Zhang $N$, Zeng $Q_{\text {, }}$ Ma $X_{1}$ et al. Diagnosis and treatment of pleuropulmonary blastoma in children: a single-center report of 41 cases. J Pediatr Surg 2019;Jun 27 [Epub]. https://doi.org/10.1016/ j.jpedsurg.2019.06.009.

10. Priest JR, McDermott MB, Bhatia S, Watterson J, Manivel JC, Dehner LP. Pleuropulmonary blastoma: a clinicopathologic study of 50 cases. Cancer 1997;80:147-61.

11. Song MS, Rossi JJ. Molecular mechanisms of Dicer: endonuclease and enzymatic activity. Biochem J 2017;474:1603-18.

12. Harris KS, Zhang Z, McManus MT, Harfe BD, Sun X. Dicer function is essential for lung epithelium morphogenesis. Proc Natl Acad Sci U S A 2006;103:2208-13.

13. Francia S, Michelini F, Saxena $A$, et al. Site-specific DICER and DROSHA RNA products control the DNA-damage response. Nature 2012;488:231-5.

14. Francia S, Cabrini M, Matti V, Oldani A, d'Adda di Fagagna F. DlCER, DROSHA and DNA damage response RNAs are necessary for the secondary recruitment of DNA damage response factors. J Cell Sci 2016;129:1468-76.

15. Burger K, Schlackow M, Potts M, Hester S, Mohammed S, Gullerova M. Nuclear phosphorylated Dicer processes double-stranded RNA in response to DNA damage. J Cell Biol 2017;216:2373-89. 